\title{
Triple-X syndrome accompanied by Chilaiditi syndrome in preterm infant: A case report
}

\author{
Yusuf Kale*, Dilek Ulubas Isik, Istemi Han Celik, Ahmet Yagmur Bas, Nihal Demirel
}

\begin{abstract}
Chilaiditi syndrome (CS) is a variant of the rotation of the colon that gives rise to the interposition of the colon between the right diaphragm and the liver intermittently or constantly. Also, Necrotizing enterocolitis (NEC) is typically seen in premature infants. In this report, we presented a female case report accompanied with a 47,XXX karyotype and CS and NEC. This association was not reported in any case study. Our findings suggest that abnormalities of X chromosome may be associated with other anomalies.
\end{abstract}

Keywords: Triple X, Chilaiditi syndrome, preterm newborn

\section{Introduction}

The triple-X syndrome is one of the most frequent aneuploid variations in female infants and the incidence of this syndrome is approximately $1 / 1000$ in population (1). A mistake in cell division called non-disjunction can result additional chromosomes in reproductive cells (2). The prevalence, like other chromosomal abnormalities increases with maternal age (3). Chilaiditi syndrome (CS) is a variant of the rotation of the colon that gives rise to the interposition of the colon between the right diaphragm and the liver intermittently or constantly. This condition occurs in $0.14 \%$ to $0.28 \%$ (4). Necrotizing enterocolitis (NEC) is typically seen in premature infants, and the timing of its onset is generally inversely proportional to the gestational age of the baby at birth (5). Here, we report an preterm infant with triple $\mathrm{X}$ accompanying $\mathrm{CS}$. To our knowledge, this is the first report of an association of these conditions.

\section{Case}

A 1.540-g female infant was born at 33 weeks gestation to a 43-year-old multigravida by cesarean delivery. Apgar scores were 7 and 8 at 1 and 5 minutes respectively. She was transferred to the neonatal intensive care unit for preterm care. Case mother had an uneventful pregnancy. There was no consanguinity between case parents. Fetal ultrasonography of case was normal. Karyotype analysis of the amniotic fluid was performed due to advanced maternal age and the triple-X syndrome was diagnosed. Prenatal diagnosis was confirmed by postnatal karyotype analysis (Figure 1). No dysmorphic features were evident at birth, and the clinical examination was normal except for mild respiratory distress. The chest X-ray revealed an elevation of the right hemi-diaphragm caused by the presence of the dilated colonic loop below (Figure 2). Chest X-ray posteroanterior view and abdomen standing reveal free gas under right dome of diaphragm. At serial interval x-rays were taken. All of them reveal free gas under right side of diaphragm only. X-rays showed a hepatodiaphragmatic interposition of the colon, leading to the diagnosis of CS. Cardiac examination revealed a grade I/VI systolic murmur and echocardiographic examination showed patent foramen ovale.

She initially made good progress and enteral feeding with breast milk was increased up to $140 \mathrm{~mL} / \mathrm{kg}$ on day 6 . On postnatal day 7 , there was an acute clinical deterioration with marked abdominal distension, vomiting and bloody stools. Oral feeding was stopped. Laboratory investigation revealed thrombocytopenia $\left(90.000 / \mathrm{mm}^{3}\right)$ and elevated C-reactive protein level. The abdomen was mildly distended without hepatosplenomegaly. Stool cultures, clostridium difficile toxin, adenovirus, norovirus and rotavirus polymerase chain reaction examinations were all negative. Stage IIB NEC was diagnosed and she was treated with intravenous fluids, antibiotics, a nasogastric tube on free drainage and bowel rest. Repeated abdominal X-ray showed intramural gas and thickened bowel walls. There was evidence of pneumoperitoneum on the X-ray 
films. A possibility of intestinal perforation was considered and surgical opinion was sought. Primary peritoneal drainage (PPD) as the initial surgical intervention for NEC was done on the $9^{\text {th }}$ day. Response to PPD was monitored by serial abdominal examinations and radiographic studies. After the return of bowel function enteral feeding was started on the 19th day and gradually increased. She made an uncomplicated recovery and was transferred back to the referring hospital on postnatal day $45^{\text {th. }}$.

\section{Discussion}

Trisomy $\mathrm{X}$ occurs from a nondisjunction event, in which the $\mathrm{X}$ chromosomes fail to properly separate during cell division (6). The first published report of a woman with a trisomy $X$ was written by Jacobs et al. (7) in 1959. Approximately 1 of every 1.000 newborn girls carries a 47,XXX karyotype (8). Most individuals with 47,XXX are diagnosed incidentally prenatal genetic screening (9). The karyotype is usually not associated with a characteristic physical phenotype. However, minor physical findings can be present in some individuals

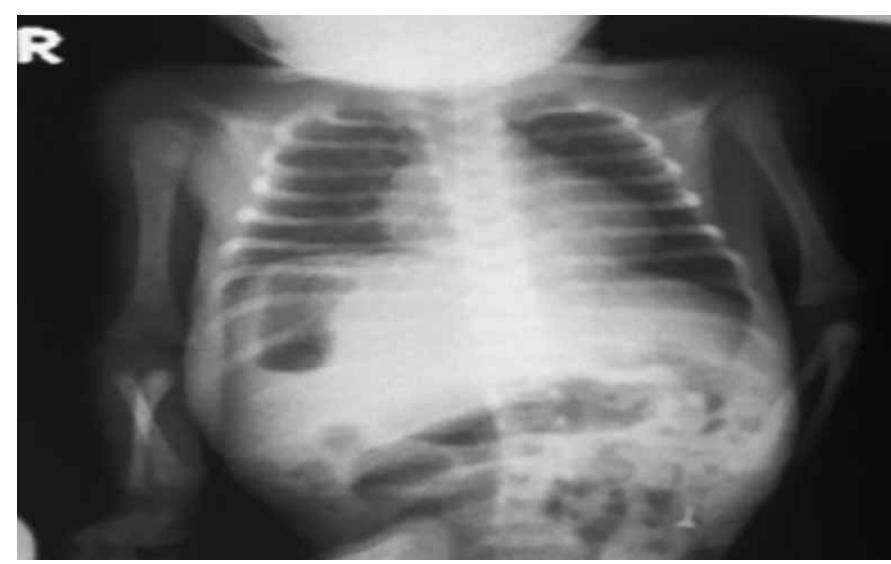

Figure 1. X-ray of the chest demonstrating elevation of the right hemi-diaphragm caused by the presence of the dilated colonic loop and gas accumulation

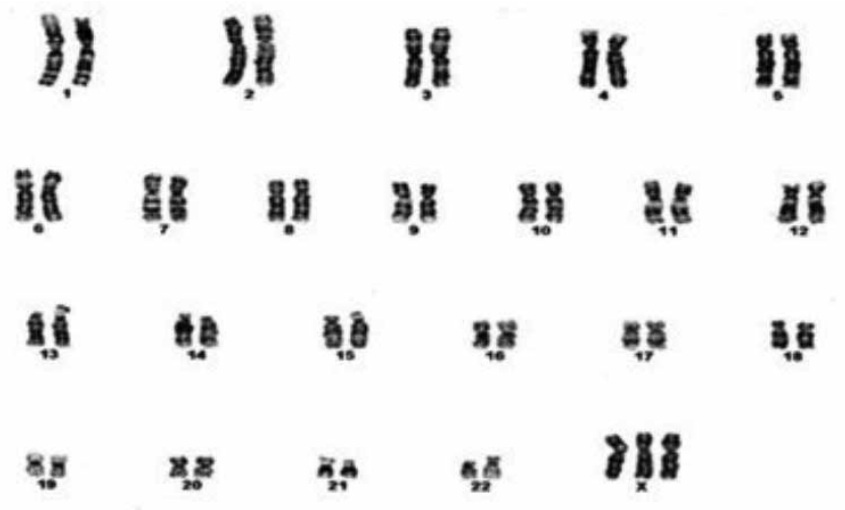

Figure 2. Patient's karyotype showing the presence of the triple $X$ as the single chromosomal aberration including epicanthal folds, hypertelorism, up-slanting palpebral fissures, clinodactyly, overlapping digits, pes planus, and pectus excavatum. Hypotonia and joint hyper-extensibility may also be present (10). Our patient had none of these physical findings. The major medical problem doesn't see in most of the cases.

Most of the previously reported cases showed an association of triple-X syndrome with urogenital malformations, ranging from unilateral kidney and renal dysplasia to ovarian malformations, and gastrointestinal abnormalities including esophageal atresia, imperforate anus, cloacal exstrophy, omphalocele and jejunal atresia (11-13). Our patient had only CS as congenital abnormality (13).

CS is a rare disorder diagnosed in childhood. This syndrome incidence increases with advancing age. Although CS is usually asymptomatic, it can lead to many several complications such as vomiting, diarrhea, volvulus, perforation, bowel obstruction, respiratory distress or cardiac arrhythmias (14). In infants, CS may be interfered with gastrointestinal abnormalities such as NEC.

In this report, we presented a female with a 47,XXX karyotype and CS and NEC. This association was not described previously. Our findings suggest that abnormalities of $\mathrm{X}$ chromosome may be associated with other anomalies.

Financial Support: This research received no specific grant from any funding agency, commercial or not-for- profit sectors.

Conflict of Interest: The authors declare no potential conflicts of interest with respect to the research, authorship, and/or publication of this article.

\section{References}

1. Nielsen J, Wohlert M. Sex chromosome abnormalities found among 34,910 newborn children: Results from a 13-year incidence study in Arhus, Denmark. Birth Defects Orig Artic Ser 1990;26:209-223.

2. Robinson A, Chapelle ADL. Sex chromosome abnormalities. In Rimoin DL, Connor JM, Pyeritz RE, editors. Emery and Rimoin's Principles and Practice of Medical Genetics. London: Churchill Living stone; 1996;3:991-992.

3. Jones KL. Smith's recognizable patterns of human malformation Recognizable Patternsof Malformation, Chromosomal Abnormality Syndromes. Philadelphia: Elsevier-Saunders Inc; 2006;6:72-73.

4. White JJ, Chavez EP, Souza J. Internal hernia of the transverse colonChilaiditi syndrome in a child. J PediatrSurg. 2002;37:802-804.

5. Lin PW, Stoll BJ. Necrotising enterocolitis. Lancet. 2006;368:12711283.

6. Hassold TJ, Hall H, Hunt P. The origin of human aneuploidy: Where we have been, where we are going. Hum Mol Genet. 2007;16:203-208. 
7. Jacobs PA, Baikie AG, Brown WM, Macgregor TN, Maclean N, Harnden DG. Evidence for the existence of the human "super female." Lancet. 1959;2:423-425.

8. Jones KL. Smith's Recognizable patterns of human malformation. Recognizable patterns of malformation, chromosomal abnormality syndromes. Philadelphia: Elsevier-Saunders Inc; 2006;6:72-73.

9. Linden $\mathrm{MG}$, Bender $\mathrm{BG}$, Robinson A. Genetic counseling for sex chromosome abnormalities. Am J Med Genet. 2002;110:3-10.

10. Linden MG, Bender BG, Harmon RJ, Mrazek DA, Robinson A. 47,XXX: What is the prognosis? Pediatrics. 1988;82:619-630.
11. Lin HJ, Ndiforchu F, Patell S. Exstrophy of the cloaca in a 47,XXX child: Review of genitourinary malformations in triple-X patients. Am J MedGenet. 1993;45:761-763.

12. Murrain L, Shanske A. Abstract: Triple X Syndrome Accompanied by Aortic Coarctation. American College of Medical Genetics Annual Meeting, Pheonix, Arizona 2008.

13. Trautner MC, Aladangady N, Maalouf E, Misra D. Jejunal atresia in an infant with triple-X syndrome. J Matern Fetal Neonatal Med. 2004;16:198-200.

14. Chang TY, Tiu CM, Chou YH. Hepatodiaphragmatic interposition of the Intestine (Chilaiditi's Syndrome). Chin J Radiol. 1999;24:101-105. 